\title{
Laser Application in Iran Urology: A Narrative Review
}

\author{
Mohammad Reza Razzaghi, Morteza Fallah Karkan, Saleh Ghiasy, Babak Javanmard* \\ Laser Application in Medical Science Research Center, Shahid Beheshti University of Medical Sciences, Tehran, Iran
}

\author{
*Correspondence to \\ Babak Javanmard MD, Assistant \\ Professor of Urology, Shahid \\ Beheshti University of Medical \\ Sciences, Tehran, Iran. \\ Tel: +982122749221 \\ Email: \\ drbabakjavanmard@gmail.com
}

Published online 26 December 2017

\section{Introduction}

The usage of laser in medicine is not recent, and the history of its use in urology goes back to 40 years ago. The first scientist who propounded the theory of 'stimulated emission' in 1917 was Albert Einstein, "the process by which photons with a certain amount of energy could disturb an excited atom and make it to drop to a lower energy level, after that, creation of another identical photon occurs. The original photon which interacts with the atom, as well as the photon which will be subsequently released, will be simultaneously discharged and will have an identical wavelength and direction of propagation."

Development of the "microwave amplification by stimulated emission of radiation" (MASER) was the first significant leap. Microwaves referred to as electromagnetic waves with fairly long wavelengths $(1 \mathrm{~mm}-1 \mathrm{~m})$. It was in 1954, the first MASER was demonstrated in an oscillator by stimulated emission at microwave wavelengths. ${ }^{2}$ it took 3 years from MASER to "light amplification by stimulated emission of radiation" (LASER). ${ }^{3}$

In 1960 Theodore Maiman succeeded to build the first LASER by using ruby crystals as active medium (chromium in corundum). The active medium in a laser defines the wavelength and frequency of the light which the laser emits and there is a reverse relationship between the wavelength and frequency. ${ }^{4}$

Gas was used as the active medium in the first lasers: carbon dioxide $(\mathrm{CO} 2)$, nitrogen $(\mathrm{N})$, neon $(\mathrm{Ne})$ and helium (He). 'Dye lasers' use liquids as active media, because the nature of the lasing agent is an organic dye. ${ }^{5}$
One of the advantages of dye lasers is the capability of generating amplified light which has a wider range of wavelengths. In 1964, one of the first solid-medium lasers was "neodymium-doped yttrium aluminium garnet" (Nd:YAG). ${ }^{6}$

One of the classifications of laser output in urology is continuous wave $(\mathrm{CW})$; the output of the laser is continuously pumped and constantly emits light; whereas in pulsed wave (PW); it releases its energy in very short pulses which can have incredibly high peak powers, that is useful for stone fragmentation.?

The pioneers of lasers in clinical urology were Parsons with a research in canine bladders in 1966, Staehler et al in bladder tumors in 1976 and Mulvany with experiments in calculi fragmentation in $1968.5,8$

For the last 2 decades, laser techniques have been widely used for management of patients with urolithiasis, bladder tumors, benign prostatic hyperplasia $(\mathrm{BPH})$, urinary tract strictures or lesions of the external genitalia.

Today, lasers broadly used in urology include Ho:YAG (holmium:YAG), Nd:YAG, CO2, Thu:YAG (thulium:YAG), LBO (lithium triborate), KTP (potassium titanyl phosphate). Due to frequent complications, the use of some types of lasers was stopped. ${ }^{2,6,7}$

Here we review all of the Iranian publications about the application of laser in urology.

\section{Methods}

This study was designed to retrieve all the studies on laser application in urology in Iran regardless of publication 
status or language, covering the years 1990-2017. Searches were carried out using Medline, Web of Science, EMBASE, Scopus, Google Scholar and Iranian databases consisting of SID, IranMedex and IranDoc (keywords: laser, Iran, urolithiasis, benign prostatic hyperplasia, bladder tumor, urethral stricture). Additionally, reference lists of identified articles deemed relevant to the review were also searched.

Twenty-six articles were identified: 12 about urolithiasis, 8 about BPH, 2 case reports, 1 paper on prostate cancer, 1 on female urethral stricture, 1 review and 1 basic sciences study.

\section{Benign Prostatic Hyperplasia}

For the last 2 decades laser techniques have been widely used for the treatment of patients with $\mathrm{BPH}$. The laser is able to ablate prostate tissue with minimal bleeding and this trait attracted urologists to use this modality to treat BPH. ${ }^{9}$ However, transurethral resection of the prostate (TURP) is still considered to be the 'gold standard' for minimally invasive management of $\mathrm{BPH} .{ }^{10}$

The Nd:YAG laser was the most studied. One of the first surgical techniques used for the treatment of $\mathrm{BPH}$ was 'visual laser ablation of the prostate' (VLAP). ${ }^{11}$ Hofstetter and Alvarez in 1993 described the interstitial laser coagulation (ILC) method with the Nd:YAG laser for management of BPH. ${ }^{12}$ Green light laser, KTP laser, PVP or photoselective vaporization of the prostate and frequencydoubled Nd:YAG laser all refer to the same modality..$^{13} \mathrm{~A}$ combination of KTP laser (to incise the bladder neck) and Nd:YAG laser ( to vaporize the prostate) named 'hybrid techniques' would decrease the neodymium-doped yttrium aluminium garnet laser's postoperative symptoms (such as irritation) and decrease time of indwelling catheter. ${ }^{14}$ The next step of prostate ablation was the 120 W LBO laser. The goal of this system was to compensate for the ability of relatively gentle tissue ablation of the 'high-level' $80 \mathrm{~W}$ KTP, which ultimates the time constant procedures in large prostate. ${ }^{15}$ The Thu:YAG laser first published in 2005, is the newest laser equipment use for $\mathrm{BPH}$ surgery. ${ }^{16}$ The latter armamentarium published with 'Thu:YAG vapo-enucleation', and ThuLEP (thulium laser enucleation of the prostate) followed them. ${ }^{17}$ Two articles published by Mahboub Ahari et al and Malek comparing PVP with TURP, showed that PVP is a safe procedure and has less complications. ${ }^{18,19}$ The holmium laser enucleation of the prostate (HoLEP) is another type of laser for BPH surgery, a comparison of this modality with TURP was published in 2011. ${ }^{20}$

Safarinezhad demonstrated that 30 of each 100000 Iranian men have BPH symptoms. Laser techniques were used in these patients as well as other surgical methods such as TURP and prostatectomy. ${ }^{21}$

The first report of laser application on $\mathrm{BPH}$ refers to Razzaghi and colleagues, who designed a randomized clinical trial study on 87 subjects in 2007 that compared laser prostatectomy versus TURP. The results showed that laser prostatectomy can reduce time being bed ridden, duration of operation and amount of fluid used in comparison with TURP and all differences were statistically significant. Also there was a remarkable difference in maximum urinary flow changes in patients whom underwent laser prostatectomy compared with TURP. ${ }^{22}$

Razzaghi et al evaluated the outcome of KTP Laser on prostate adenomas on 40 patients in 2010. They reported that in pathologic samples, 0 to $2 \mathrm{~mm}$ of tissue destruction with the disappearance of nuclei of the cells, basophilia of the stroma, and damaged tissue (cutter like effect) were witnessed. Findings were compatible with burn effect. The authors concluded that KTP laser prostatectomy has lower risk of complications such as perforation of capsule and extra penetration of prostate tissue during the procedure. ${ }^{23}$

Razzaghi et al reported treatment of human prostates by diode laser in 70 patients with BPH in 2012. This study explained that $100 \mathrm{~nm} 980 \mathrm{~W}$ diode laser vaporization prostatectomy was achievable and appeared to be unharmful and efficient for the treatment of bladder outlet obstruction. During surgery by diode laser, no intraoperative complications, fluid absorption or blood loss occurred. Important improvements after surgery include increase in Qmax, reductions in international prostate scoring system, and decrease in post-void residual (PVR) urine and there was significant decrease in PSA level and prostate volume after PVP at time of follow-up. ${ }^{24}$

In a randomized clinical trial on 115 cases with $\mathrm{BPH}$ that was published by Razzaghi et al in 2014 comparing diode laser $(980 \mathrm{~nm})$ vaporization with TURP. Diode laser was suggested as a safe procedure in the treatment of $\mathrm{BPH}$; but, at longer time of follow-up, the efficacy of diode laser vaporization was inferior to TURP. In comparison of the TURP and diode groups, Foley catheterization time and postoperative hospitalization in the diode group was lower than for TURP $(P=0.0001)$. In the TURP group, capsule perforation, need for blood transfusion, and TUR syndrome were seen, whereas these complications were not observed in the other group. ${ }^{25}$

In a study in 2013, Iman Talab et $\mathrm{al}^{26}$ reported the management of anesthesia in a 69 years old patient with bladder rupture during prostate resection with laser diode $980 \mathrm{~nm}$. Advised anesthesiologic approaches in the literature for such situations are (1) early diagnosis of bladder rupture, (2) spinal anesthesia instead of general anesthesia, (3) stop the surgery, (4) stop N2O halation in general anesthesia, and (5) execution of academic principles of surgical technique.

Laser advantages include reduced risk of bleeding, possibility to use saline for washing, reduced absorption of fluids and reduced inflammation, the possibility of general anesthesia and sedation for most patients as well 
as the ability to continue taking anticoagulant in patients at risk of thromboembolic complications. ${ }^{26}$

\section{Treatment of Urolithiasis With Laser}

Renal stones treatment shifted from open surgery to less invasive interventions over the last decades. Endoscopic laser lithotripsy is commonly used as a treatment for patients with urolithiasis. The first paper about pulsed dye laser for treatment of urolithiasis was presented in $1987 .{ }^{27}$ The next one was the "frequency doubled double pulse Nd:YAG” (FREDDY), including a KTP crystal embedded into a Nd:YAG laser. ${ }^{28}$ A number of lasers have been trialed for laser lithotripsy, of which, the Ho:YAG has become one of the most commonly used. ${ }^{29}$ The mechanism of laser stone fragmentation is through photothermal effect, which depends on contact of the laser tip with the stone. ${ }^{30}$ A major benefit is least retropulsion effect in the course of surgery. ${ }^{31}$

In a prospective randomized controlled trial, in 2008, Maghsoudi et al studied 79 subjects with 82 ureteral calculi, comparing Ho:YAG laser vs pneumatic lithotripsy. The retropulsion effect occurred in 1 case from the laser group $(2.4 \%)$ vs 3 cases of pneumatic lithotripsy $(P=0.6)$. Stone free rate (SFR) was higher in Ho:YAG laser group vs pneumatic lithotripsy (37 cases $(90.2 \%)$ vs 30 cases (73.20\%) $(P=0.46){ }^{32}$

In another randomized controlled trial, in 2010, Basiri et al studied 100 children with distal ureteral stone, compared transurethral lithotripsy with shock wave lithotripsy (SWL) and holmium laser to evaluate the superior modality. Both were suitable modalities for removal of distal ureteral stone at that age range. SFR was remarkably higher in the transurethral lithotripsy group than in the SWL group (respectively $P<0.004$ and $P<0.001)$ and need to repeat the treatment in SWL was higher than transurethral lithotripsy (TUL) $(P<0.004) .^{33}$ In a case report, Hosseini et al in 2011 presented a patient with a solid stony mass which was in the perineum after Two-Stage Urethroplasty. They concluded that Ho: YAG laser is a minimally invasive modality which is suitable for management of urethral stones in such cases. ${ }^{34}$

Amjadi et al in 2011 published a report of transurethral lithotripsy of ureteral stone with holmium in 41 children $\leq 12$ years. They demonstrated that complications after ureteroscopic laser lithotripsy are rare. With the application of Ho: YAG laser with flexible fibers, the chance of treatment increases and SFR was $89.35 \%{ }^{35}$

In another study, Razzaghi et al compared pneumatic and holmium laser on 112 patients in 2011. They reported that laser lithotripsy is an advanced method for treatment of ureteral stones larger than $1 \mathrm{~cm}$. The success rate in the Ho: YAG laser group was higher than in the pneumatic group $(P=0.003)$. Operation time and hospitalization time were decreased, and there were no significant complications. ${ }^{36}$

In a case report published by Razzaghi et al, the use of
Ho: YAG laser ureteroscopic lithotripsy in a patient with ectopic malrotated kidney was discussed, and Ho: YAG laser lithotripsy was effective and safe in this patient. ${ }^{37}$ In a randomized clinical trial done in 2013 by Razzaghi et al on 112 subjects, pneumatic lithotripters and holmium laser were compared in the treatment of ureteral stones. Laser lithotripsy was safer and a more effective procedure for the treatment of upper ureteral calculi with a diameter of 1 to $2 \mathrm{~cm}$, due to its higher SFR. SFR in the laser group was higher than in the pneumatic group $(P=0.001) .{ }^{38}$ Javanmard et al in 2015 compared flexible ureteroscopy versus extracorporeal SWL (ESWL) in the treatment of renal stones of $1-2 \mathrm{~cm}$ in 58 obese patients. Retrograde intrarenal surgery (RIRS) offers a good outcome for treatment of these patients. The operation time, SFR and rate of complication were higher in RIRS group vs ESWL group $\left(P=0.061, P=0.019\right.$ and $P=0.211$, respectively).$^{39}$ In another randomized clinical trial by Javanmard et al on 46 patients with renal stones in 2016, they compared outcomes of RIRS with ESWL for stones $\leq 2 \mathrm{~cm}$. The RIRS procedure was associated with less pain and higher success and was a safe option for renal stones $\leq 2 \mathrm{~cm}$. Inferior calyx stones resolved significantly in RIRS vs SWL $(100 \%$ vs. $40 \%, P=0.02) .{ }^{40}$

Mehrabi et al studied 59 patients for comparison of laser lithotripsy by semi-rigid ureteroscope vs ESWL. SFR in semi-rigid ureteroscope vs ESWL was $85 \%$ and $87.5 \%$ respectively $(P>0.05)$. They concluded that transurethral lithotripsy by semi-rigid ureteroscope can be used as an alternative modality in management of upper ureteral calculi. ${ }^{41}$

In a prospective study by Karami et al, 48 patients were assessed for the safety and efficacy of transurethral cystolithotripsy of large bladder stones by holmium laser in the outpatient setting. SFR was 98.5\%. Major complications and recurrence of bladder stone were not seen. ${ }^{42}$

Another study was about the safety and efficacy of laser lithotripsy in pregnancy reported by Abedi et al. In this retrospective study, 15 pregnant women with ureteral stones treated with semi-rigid ureteroscope and holmium laser were included. They concluded that laser lithotripsy is a safe method in pregnant patients with ureteral stone where conservative treatment failed. ${ }^{43}$

\section{Lower and Upper Urinary Tract Strictures}

The description of an argon laser for treatment of urethral strictures goes back to 1978. Using the laser instead of a cold knife, ${ }^{44}$ looked effective, however follow up demonstrated a recurrence rate of up to $70.1 \%$ in a mean time of 15.2 months. Ho:YAG, Nd:YAG and KTP lasers have also been investigated. ${ }^{45}$ Recently, the Thu:YAG laser was investigated in this setting. Penetration depth of this laser which is only $0.3 \mathrm{~mm}$ leads to very limited injury to adjacent tissue. ${ }^{46}$ The Ho:YAG laser can treat ureteropelvic junction obstruction (UPJO) with the 
endopyelotomy method, similar outcomes to those with the 'hot-wire balloon' were achieved..$^{30}$ The Ho:YAG laser is also effective in the minimally invasive management of ureterointestinal anastomotic strictures after urinary diversion as well as ureterovesical strictures at anastomosis sites after renal transplant. ${ }^{47}$

Razzaghi reported a 19-year-old male patient with urethral stricture that underwent internal urethrotomy with Ho-YAG laser. Total of fibrotic tissues was cut and a $18 \mathrm{Fr}$ siliconized Foley catheter passed for the patient. According to this study, urethrotomy with Ho-YAG laser is less invasive and safe. It is effective alternative for patients taking anti-coagulant drugs and are prone to have urethral bleeding. ${ }^{48}$

In a study, Razzaghi et al presented a 47-year-old woman who had undergone previous open pyeloplasty. Just below the right UPJ, a full-thickness lateral incision was done with Ho:YAG laser and carried through the stenotic segment up to the renal pelvis. Three months after surgery, half-time of excretion of radioisotope from right kidney in diuretic renogram was 13 minutes. Complications such as bleeding, pain, leakage and infection were not reported. ${ }^{49}$ In a prospective study, Sharifiaghdas et al incised bladder neck with 2-micron CW laser in a woman with bladder outlet stricture. After treatment, improvement of International Prostate Symptom Score (IPSS) $(P=0.005)$, quality of life $(P=0.005)$ and reduction of $\operatorname{PVR}(P=0.003)$ were seen. ${ }^{50}$

\section{Prostate Adenocarcinoma}

The probability of focal laser ablation of localized prostate cancer has recently been studied. The diode laser or Nd:YAG laser can be used for this purpose. A recent advance is the ability of real-time monitoring of lesion created by the laser with magnetic resonance imaging. ${ }^{51}$ In a study in 2014, Javanmard et al investigated 36 patients to evaluate laser ablation of prostate by diode laser prostate for treating bladder outlet obstruction symptoms due to prostate cancer. After surgery, the results of the comparison between diode laser ablation and channel TUR of prostate showed improving IPSS, PVR and Qmax in both groups. Mean IPSS was $11.1 \pm 4.1$ in TURP group vs $11.7 \pm 3.6$ in laser group $(P=0.64)$. Mean PVR was 18.4 $\pm 3.5 \mathrm{~mL}$ in TURP group vs $17.7 \pm 6.3 \mathrm{~mL}$ in laser group $(P=0.68)$. Mean Qmax in TURP vs laser groups was 20.1 $\pm 4.5 \mathrm{~mL} / \mathrm{s}$ vs $19.4 \pm 2.6 \mathrm{~mL} / \mathrm{s}$, respectively $(P=0.57) .{ }^{52}$

\section{Seminiferous Epithelium}

In a basic study, Fakhataha et al in 2003 investigated low power gallium-aluminium-arsenide $(830 \mathrm{~nm})$ laser to determine the qualitative and ultrastructural changes of the seminiferous epithelium after laser radiation. Laser radiation can lead to double contradictory effects on spermatogenesis. Low doses of laser lead to improvement in the count of the germ cells with normal ultrastructural features, while the doses higher than biostimulative threshold can lead to defect in spermatogenesis. ${ }^{53}$

\section{Conclusion}

We reviewed all the available literature on laser application in Iran. Although laser applications in medical science and especially urology are at an increasing pace, we conclude that usage of this technology has not yet found its position in Iran, especially in the field of urology. The main causes for this are the difficult accessibility and disturb of laser devices and its accessories, as well as the lack of adequate knowledge of the medical community about this modality. As mentioned in the above review, the published studies have mostly been from a university center that has been the founder and practitioner of laser applications in medical sciences and the field of urology, which indicates a weakness in extending the use of this technology in universities.

On the basis of the literature review in this paper future work will involve:

- Put training of laser foundations and its applications in medical sciences in specialized curriculum training

- Holding courses and workshops

- Organizing congresses and live surgery/performances or video

- Conducting laser medical rehearsal courses

- Require learning of some operation, such as ureteral stone or prostate laser in Urology educational curriculum.

\section{Conflict of Interests}

The authors declare that they have no conflict of interest.

\section{Ethical Considerations}

Not applicable.

\section{References}

1. Einstein A. Zur Quantentheorie der Strahlung. Phys Z. 1917;18:121-128.

2. Gordon JP, Zeiger HJ, Townes CH. Molecular microwave oscillator and new hyperfine structure in the microwave spectrum of NH3. Phys Rev. 1954;95(1):282-284.

3. Hecht J. A short history of laser development. Appl Opt. 2010;49(25):F99-122. doi:10.1364/ao.49.000f99

4. Maiman TH. Stimulated optical radiation in ruby. Nature. 1960;187:493-494. doi:10.1038/187493a0

5. Gross AJ, Herrmann TR. History of lasers. World J Urol. 2007;25(3):217-220. doi:10.1007/s00345-007-0173-8

6. Geusic JE, Marcos HM, Van Uitert LG. Laser oscillations in Nd-doped yttrium aluminum, yttrium gallium, and gadolinium garnets. Appl Phys Lett. 1964;4(10):182-184. doi:10.1063/1.1753928

7. Teichmann HO, Herrmann TR, Bach T. Technical aspects of lasers in urology. World J Urol. 2007;25(3):221-225. doi:10.1007/s00345-007-0184-5

8. Staehler G, Hofstetter A, Gorisch W, Keiditsch E, Mussiggang M. Endoscopy in experimental urology using an argon-laser beam. Endoscopy. 1976;8(1):1-4. doi:10.1055/s-0028-1098365 
9. Anson K, Seenivasagam K, Miller R, Watson G. The role of lasers in urology. Br J Urol. 1994;73(3):225-230. doi:10.1111/j.1464-410X.1994.tb07509.x

10. Sarf I, Thuroff J, Cheng C, Gross A, Kuntz R. Plenery on male voiding dysfunction. Société Internationale D'Urologie (SIU) World Meeting on Lower Urinary Tract Dysfunction' Marrakech, Morocco: 2010:13-16.

11. Norris JP, Norris DM, Lee RD, Rubenstein MA. Visual laser ablation of the prostate: clinical experience in 108 patients. J Urol. 1993;150(5 Pt 2):1612-1614.

12. Hofstetter A, Alvarez A. Treatment of prostatic tumors with interstitial thermocoagulation with neodymiumYAG (a new treatment in minimally invasive surgery). Arch Esp Urol. 1993;46(4):317-319.

13. McAllister WJ, Gilling PJ. Vaporization of the prostate. Curr Opin Urol. 2004;14(1):31-34.

14. Barber NJ, Muir GH. High-power KTP laser prostatectomy: the new challenge to transurethral resection of the prostate. Curr Opin Urol. 2004;14(1):21-25.

15. Wosnitzer MS, Rutman MP. KTP/LBO laser vaporization of the prostate. Urol Clin North Am. 2009;36(4):471-483, vi. doi:10.1016/j.ucl.2009.08.004

16. Xia SJ, Zhang YN, Lu J, et al. [Thulium laser resection of prostate-tangerine technique in treatment of benign prostate hyperplasia]. Zhonghua Yi Xue Za Zhi. 2005;85(45):3225-3228.

17. Bach T, Xia SJ, Yang Y, et al. Thulium:YAG $2 \mathrm{~mm} \mathrm{cw}$ laser prostatectomy: where do we stand? World J Urol. 2010;28:163-168.

18. Mahboub-Ahari A, Sadeghi-Ghyassi F, Yousefi M, Amjadi M, Mostafaie A. Green light photo selective vaporization of the prostate vs. transurethral resection of prostate for benign prostatic hyperplasia. J Lasers Med Sci. 2011;2(4):152-158.

19. Malek R. Photoselective prostate evaporation with KTP or PVP laser. Med Laser J. 2008;5(3 and 4):1-3.

20. Hajebrahimi S, Mahboub-Ahari A, Sadeghi-Ghyassi F, Mostafaie A, Yousefi M. Holmium Laser Prostate Enucleation (HOLEP) Versus Trans-Urethral Resection of Prostate (TURP) in Treatment of Symptomatic Prostatic Enlargement; A Health Technology Assessment. J Laser Med Sci. 2011;2(1):36-42. doi:10.22037/2010.v2i1.2159

21. Safarinejad MR. Prevalence of benign prostatic hyperplasia in a population-based study in Iranian men 40 years old or older. Int Urol Nephrol. 2008;40(4):921-931. doi:10.1007/ s11255-008-9338-7

22. Razzaghi MR, Habibi G, Djavid GE, Gholamrezaee H. Laser prostatectomy versus transurethral resection of prostate in the treatment of benign prostatic hyperplasia. Saudi Med J. 2007;28(1):68-72.

23. Razzaghi MR, Hosseini MM, Rezaei A, Rezaei I, Javanmard B, Mazloomfard MM. Effect of KTP Laser on Surgically Resected Adenomas of Prostate. J Lasers Med Sci. 2010;1(1):20-23. doi:10.22037/2010.v1i1.2133

24. Razzaghi MR, Mokhtarpour H, Mazloomfard MM. Diode laser treatment of human prostates. J Lasers Med Sci. 2012;3(1):1-5. doi:10.22037/2010.v3i1.2625

25. Razzaghi MR, Mazloomfard MM, Mokhtarpour H, Moeini A. Diode laser $(980 \mathrm{~nm})$ vaporization in comparison with transurethral resection of the prostate for benign prostatic hyperplasia: randomized clinical trial with 2-year follow-up. Urology. 2014;84(3):526-532. doi:10.1016/j. urology.2014.05.027

26. Iman-Talab V, Haghighi M, Sedighinejad A, Naderi nabi B, karami M-S, Jamali K. Anesthesia management in a patient with bladder perforation during TURP with $980 \mathrm{~nm}$ diode laser: A case report. Journal of Anesthesiology and Pain. 2015;4(3):69-74. [Persian].

27. Dretler SP, Watson G, Parrish JA, Murray S. Pulsed dye laser fragmentation of ureteral calculi: initial clinical experience. J Urol. 1987;137(3):386-389.

28. Marks AJ, Teichman JM. Lasers in clinical urology: state of the art and new horizons. World J Urol. 2007;25(3):227233. doi:10.1007/s00345-007-0163-x

29. Lee J, Gianduzzo TR. Advances in laser technology in urology. Urol Clin North Am. 2009;36(2):189-198, viii. doi:10.1016/j.ucl.2009.02.004

30. Pierre S, Preminger GM. Holmium laser for stone management. World J Urol. 2007;25(3):235-239. doi:10.1007/s00345-007-0162-y

31. Cinman NM, Andonian S, Smith AD. Lasers in percutaneous renal procedures. World J Urol. 2010;28(2):135-142. doi:10.1007/s00345-009-0423-z

32. Maghsoudi R, Amjadi M, Norizadeh D, Hassanzadeh H. Treatment of ureteral stones: A prospective randomized controlled trial on comparison of Ho:YAG laser and pneumatic lithotripsy. Indian J Urol. 2008;24(3):352-354. doi:10.4103/0970-1591.39549

33. Basiri A, Zare S, Tabibi A, et al. A multicenter, randomized, controlled trial of transureteral and shock wave lithotripsy-which is the best minimally invasive modality to treat distal ureteral calculi in children? J Urol. 2010;184(3):11061109. doi:10.1016/j.juro.2010.05.021

34. Hosseini SJ, Mokhtarpour H, Bagher-Tabrizi A, Hasanzadeh Hadad A. Urethroscopic holmium: yag laser ablation of large urethral stone after two-stage urethroplasty. J Lasers Med Sci. 2011;2(2):87-88. doi:10.22037/2010.v2i2.2289

35. Amjadi M, Kazemi Rashed F, Sari Motlagh R, Sheikh Monazzah F. Transureteral lithotripsy of ureteral calculi in children with Holmium:Yttrium Aluminium Garnet (YAG ) laser: J Lasers Med Sci. 2011;2(4):148-151. doi:10.22037/2010.v2i4.2624

36. Razaghi MR, Razi A, Mazloomfard MM, Mokhtarpour $H$, Javanmard B, Mohammadi R. Trans-ureteral ureterolithotripsy of ureteral calculi: which is the best; pneumatic or holmium laser technique? J Lasers Med Sci. 2011;2(2):59-62.

37. Razzaghi MR, Mazloomfard MM, Mohammadi R, Bahrami-Motlagh H. Holmium-YAG Laser Ureteroscopic Lithotripsy in a Patient with Ectopic Malrotated Kidney. J Lasers Med Sci. 2012;3(2):75-78. doi:10.22037/2010. v3i2.2850

38. Razzaghi MR, Razi A, Mazloomfard MM, Golmohammadi Taklimi A, Valipour R, Razzaghi Z. Safety and efficacy of pneumatic lithotripters versus holmium laser in management of ureteral calculi: a randomized clinical trial. Urol J. 2013;10(1):762-766.

39. Javanmard B, Razaghi MR, Ansari Jafari A, Mazloomfard MM. Flexible Ureterorenoscopy Versus Extracorporeal Shock Wave Lithotripsy for the Treatment of Renal Pelvis Stones of 10-20 mm in Obese Patients. J Lasers Med Sci. 2015;6(4):162-166. doi:10.15171/jlms.2015.12

40. Javanmard B, Kashi AH, Mazloomfard MM, Ansari Jafari 
A, Arefanian S. Retrograde Intrarenal Surgery Versus Shock Wave Lithotripsy for Renal Stones Smaller Than 2 cm: A Randomized Clinical Trial. Urol J. 2016;13(5):28232828 .

41. Mehrabi S, Rahmani A, Mehrabi A, Motlagh A. Extracorporeal shockwave lithotripsy versus laser lithotripsy by semirigid ureteroscope in treatment of upper ureteral stones. Acta Med Mediter. 2016;32:2075.

42. Karami H, Razaghi MR, Javanmard B, et al. Outpatient Transurethral Cystolithotripsy of Large Bladder Stones by Holmium Laser. J Lasers Med Sci. 2016;7(1):12-15. doi:10.15171/jlms.2016.03

43. Abedi AR, Allameh F, Razzaghi MR, et al. The Efficacy and Safety of Laser Lithotripsy in Pregnancy. J Lasers Med Sci. 2017;8(2):84-87. doi:10.15171/jlms.2017.15

44. Bagley DH, Grasso M, 3rd. Ureteroscopic laser treatment of upper urinary tract neoplasms. World $J$ Urol. 2010;28(2):143-149. doi:10.1007/s00345-010-0525-7

45. Pinthus JH, Bogaards A, Weersink $\mathrm{R}$, Wilson BC, Trachtenberg J. Photodynamic therapy for urological malignancies: past to current approaches. J Urol. 2006;175(4):1201-1207. doi:10.1016/s00225347(05)00701-9

46. Sachse H. [Direct vision cold knife internal urethrotomy (author's transl)]. Urologe A. 1978;17(3):177-181.

47. Becker HC, Miller J, Noske HD, Klask JP, Weidner W. Transurethral laser urethrotomy with argon laser: experience with 900 urethrotomies in 450 patients from
1978 to 1993. Urol Int. 1995;55(3):150-153.

48. Razaghi MR, Mazloomfard MM, Javanmard B, Mohammadi R, Ansari Jafari A. Direct vision internal urethrotomy with application of holmium:YAG laser: $J$ Lasers Med Sci. 2011;2(3):126-128.

49. Razaghi MR, Razi A, Mokhtarpour H, Mazloomfard MM, Lotfi B.Retrograde Holmium- YAG Laser Endopyelotomy. J Laser Med Sci. 2011; 2(1):46-48. doi:10.22037/2010. v2i1.2156

50. Sharifiaghdas F, Kardoust Parizi M, Ahadi B. Efficacy of Transurethral Bladder Neck Incision with 2-Micron Continuous Wave Laser (RevoLix) for the Management of Bladder Outlet Stricture in Women. Urol J. 2013;10(1):790794.

51. Wang L, Wang Z, Yang B, Yang Q, Sun Y. Thulium laser urethrotomy for urethral stricture: a preliminary report. Lasers Surg Med. 2010;42(7):620-623. doi:10.1002/ lsm.20934

52. Javanmard B, Hassanzadeh Haddad A, Yaghoobi M, Lotfi B. Diode laser ablation of prostate and channel transurethral resection of prostate in patients with prostate cancer and bladder outlet obstruction symptoms. Urol J. 2014;11(4):1788-1792.

53. Fakhataha $M$, Velojerdi $M$, Altarihi T. Investigation of Low-Power Galium-Aluminum-Arsenide (830 nm) Laser Radiation Effects on Qualitative and Ultra-Structural Properties of semineferuos tubule Epithelium. Anat Sci J. 2003;1(3):27-38. 